\title{
MPPT Control Method for Photovoltaic System Based on Particle Swarm Optimization and Bacterial Foraging Algorithm
}

\author{
Zhiguo Zhu, Guowei Liu* \\ Department of Electrical and Information Engineering, Anhui University of Science and Technology, Huainan, China \\ Email address: \\ gwliu@aust.edu.cn(GuoweiLiu) \\ ${ }^{*}$ Corresponding author \\ To cite this article: \\ Zhiguo Zhu, Guowei Liu. MPPT Control Method for Photovoltaic System Based on Particle Swarm Optimization and Bacterial Foraging \\ Algorithm. International Journal of Electrical Components and Energy Conversion. Vol. 4, No. 1, 2018, pp. 45-49. \\ doi: 10.11648/j.ijecec.20180401.15
}

Received: April 12, 2018; Accepted: April 27, 2018; Published: May 19, 2018

\begin{abstract}
The P-V output feature of photovoltaic (PV) array presents multi-wave peaks under non-uniform illumination, so the traditional algorithm can not overcome the shortcomings of the local optimal value. In this paper, an optimization algorithm based on particle swarm and bacteria foraging is proposed, which is applied to the maximum power point tracking (MPPT) of PV arrays. The algorithm introduces the tendency operation to find the optimal solution in the local range. The replication operation is introduced to avoid the blind randomness of population update, and the convergence speed of the algorithm is accelerated. The migration operation is introduced to avoid the algorithm falling into the local optimal solution. The output power characteristics of PV array under occlusion are analyzed, and the MPPT control method experiment is carried out using bacterial foraging algorithm (BFA). Experimental results show that the algorithm can get rid of the constraint of local optimal value, quickly find the global maximum power point, and the control precision is high. It provides a new implementation method for PV array MPPT.
\end{abstract}

Keywords: PV Array, MPPT, PSO, BFA, Partial Shadow

\section{Introduction}

At present, solar energy has been widely used for its cleanness and reproducibility [1,2]. In the case of partial shadow, the power loss of PV array can be as high as $70 \%$. Due to the existence of occlusion, the illumination of the whole PV array is not uniform, resulting in multiple peaks on the P-V curve, and the traditional MPPT algorithm falls into the local peak. The real global maximum power points could not be found, resulting in the failure of these algorithms. To solve this problem, a heuristic algorithm is proposed, such as PSO algorithm, BFA algorithm, immune algorithm and so on $[3,4]$. The combination of BFA and PSO is introduced into the MPPT algorithm. BFA tends to make the local search ability stronger, but because BFA bacteria have no memory ability, its global search ability is weakened. Since PSO particles have memory properties for individual and group optimal information. PSO algorithm is introduced into BFA and then a new PSO-BFA algorithm is obtained [5]. PSO algorithm is used to complete global space search. Particle is used as local operation of BFA. This combines the global search capability of PSO and the local search capability of BFA. PSO only uses population search part BFA uses PSO memory function to improve global search capability and search efficiency [6]. According to the rule of PV array partial shadow under the condition of partial shadow. Bacteria is distributed near the possible extremum under initial conditions to avoid falling into local extremum. The efficiency of the algorithm is improved effectively. 


\section{Application of PSO-BFA in Multi-peaks}

Particle swarm-bacterial foraging algorithm is based on BFA and combines PSO with strong global search algorithm. BFA is a bionic intelligent algorithm based on Escherichia coli in human intestinal tract of 2002. Bacteria foraging has the behavior of chemotaxis, reproduction, elimination and dispersal [7]. Bacteria foraging tendency operation makes its local search ability stronger but bacterial foraging ability can only be achieved by migration, the but global search ability is limited by migration probability $\mathrm{P}_{\text {ed }}$. PSO has the characteristics of global strength, individual effect and group effect. Therefore, I get particle swarm-bacterial foraging algorithm by combining these two algorithms [8].

\section{1. $B F A$}

Three operations of BFA abstracted from bacteria foraging behavior are shown in Figure 1. In the figure, $N_{e d}$ represents maximum number of migration operations. $N_{r e}$ represents maximum number of replication operations. $N_{c}$ represents maximum times of trend operations and $l, k, j$ respectively represent $l$-migration, $k$-replication and $j$-tendency operations.

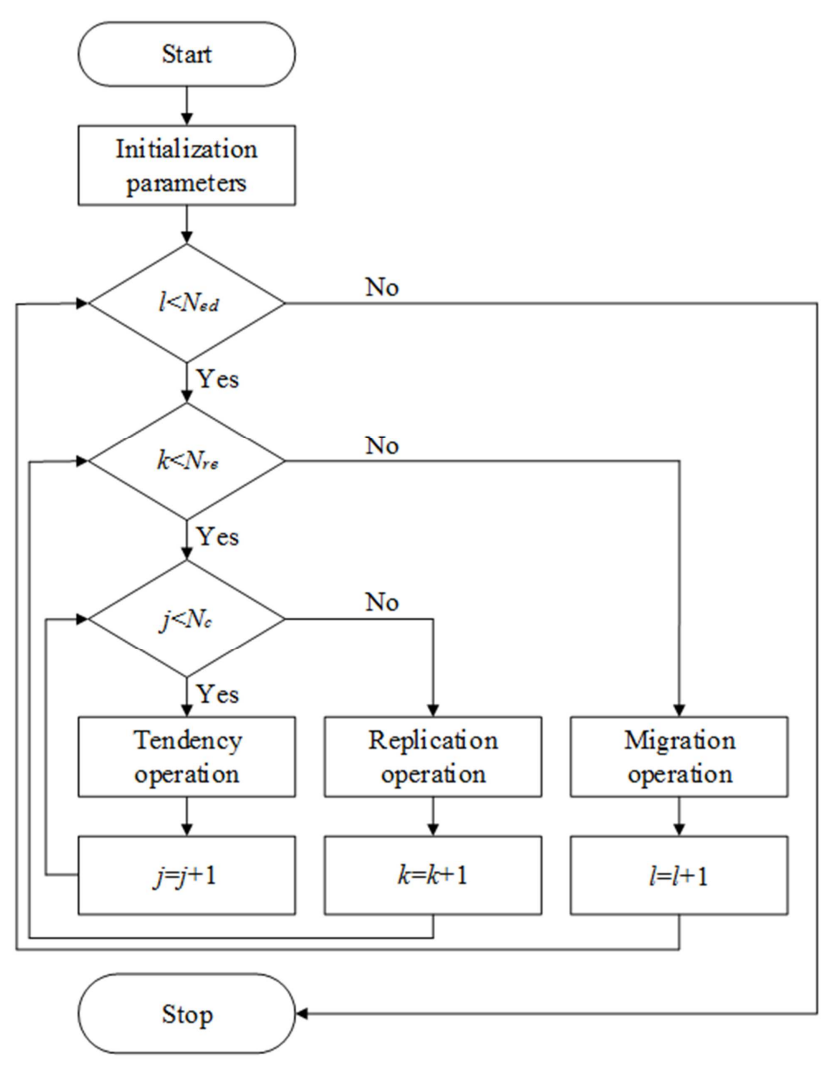

Figure 1. Flow of BFA.

(1) Tendency operation: In areas where the environment is poor, bacteria rotate frequently, while in areas where the environment is better, bacteria tend to swim more. The whole life cycle of Escherichia coli is to change between the two basic movements of rotation and swimming [9]. Rotation and swimming give bacteria a better living environment. This behavior of bacteria moving to rich areas is called tendency behavior. The size of the bacterial population is $S$, in which each bacterium is represented by $\theta^{i}$, and then $\theta^{i}(j, k, l)$ represents the position of bacterial $i$ after the $j$-tendency operation, the $k$-replication operation, and the $l$-migration operation. Each step tendency operation of bacterial $i$ represents is as follows:

$$
\theta^{i}(j+1, k, l)=\theta^{i}(j, k, l)+c(i) f(j)
$$

Among them $c(i)$ is a positive step to determine direction. Tendency operations are bacteria locally searched because PV plates are 2-D curves. So there are only two directions when the bacteria move in the direction of movement. When they move in one direction, the power in the direction of rotation decreases, $f(j)$ represents the random direction of forward selection after rotation.

The tendency operation makes BFA have the ability of local mining, which determines the direction of the algorithm and the degree of search details in a certain area. It is the core operation of BFA. This is also an important part of the algorithm design. When applied to MPPT, the direction of motion is limited to two dimensional space, but the voltage does not increase or decrease $[10,11]$. In order to improve the search speed of the algorithm, when the fitness of the new position becomes worse after the bacteria rotates, it will move in the opposite direction until it reaches a certain step size or the new fitness is no longer improved.

(2) Replication operation: Evolutionary theory shows that biological evolution is a process of survival of the fittest. After a period of food search, some of the weaker bacteria that look for food will be eliminated naturally, and the remaining bacteria will multiply because they are well nourished. This phenomenon is called replication behavior in BFA. BFA was copied by elite retention. The replication operation selected the half of bacteria $S / 2$ with weak survivability to be eliminated, and the other half with strong survival ability were used to reproduce the new generation. By self-replicating, each of them produces a new individual that is exactly the same as himself. So that the high-quality bacterial individuals can lead the population to multiply, thus improving the global convergence and computational efficiency. After replication, the population size remains the same.

(3) Migration operation: Mutations may occur in the local area of bacterial life, leading to the collective migration of bacterial populations to a new local area. The behavior of simulates this phenomenon in the BFA is called migration behavior. Migration occurs with a certain probability $\mathrm{P}_{\text {ed }}$. If a bacterial individual in the population satisfies the probability of migration, the bacteria die out and randomly generate a new individual at any location. This new individual may have a different position than the perishing individual. The migration operation makes BFA have the ability of random search, which is helpful for BFA to keep the diversity of population, reduce the situation of premature convergence, and help the tendency operation jump out of the local optimal 
solution and find the global optimal solution. Because the MPPT requires faster convergence, the migration probability can not be too high.

\subsection{PSO}

The flow of PSO is shown in Figure 2. PSO is a kind of bionic algorithm for food search of natural birds. Birds are regarded as a particle in space, and bird swarm is a kind of particle swarm. Each particle itself carries the corresponding information, such as its own velocity and position, and determines the distance and direction of its motion according to the corresponding information of the particle itself. PSO is to initialize a group of particles randomly distributed into a solution space to be searched, and then iterate according to the given formula. The formula of PSO contains two optimal concepts, a local optimal $p_{\text {best }}$ and a globally optimal $g_{\text {best }}$. The local optimum is the best solution obtained by each particle in the search process, and the global optimization is the optimal solution obtained by the particle swarm. This PSO algorithm has the characteristics of memory, the use of positive feedback adjustment, the principle of the algorithm is simple, few parameters and good applicability. The formula of PSO is as follows:

$$
\begin{gathered}
v_{i d}^{k+1}=w v_{i d}^{k+1}+c_{1} \operatorname{rand}_{1}^{k}\left(p_{\text {best }}-x_{i d}^{k}\right)+c_{2} \operatorname{rand}_{2}^{k}\left(g_{\text {best }}-\right. \\
\left.x_{i d}^{k}\right) \\
x_{i d}^{k+1}=x_{i d}^{k}+v_{i d}^{k+1}
\end{gathered}
$$

Where, $v$ denotes velocity, $k$ is the number of iterations, $c_{1}$ and $c_{2}$ are learning factors. rand $_{1}$ and rand $_{2}$ are random numbers between $[0,1] . x_{i d}$ denotes location and $p_{\text {best }}$ denotes local optimum; $g_{\text {best }}$ represents global optimality.

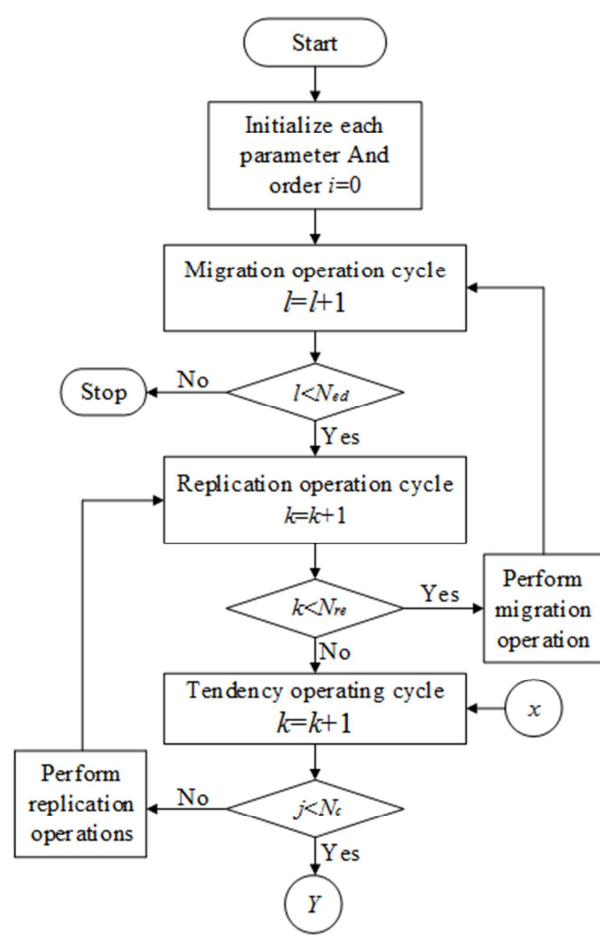

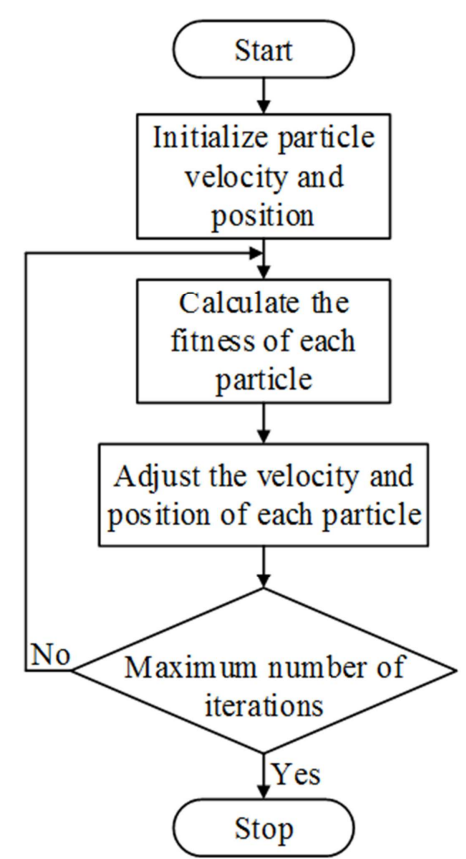

Figure 2. Flow of PSO.

\subsection{PSO-BFA}

Although the BFA can change its direction and improve its local search accuracy, but the bacterial global search can only rely on migration operation. Therefore, its global search ability is weak. Because PSO has the ability of memory and global search, the particle is used for global search, the particle is regarded as bacteria, and the BFA is used for the tendency operation. Thus, the global searching ability of BFA is improved. The flow of particle swarm-bacteria foraging algorithm is shown in Figure 3.

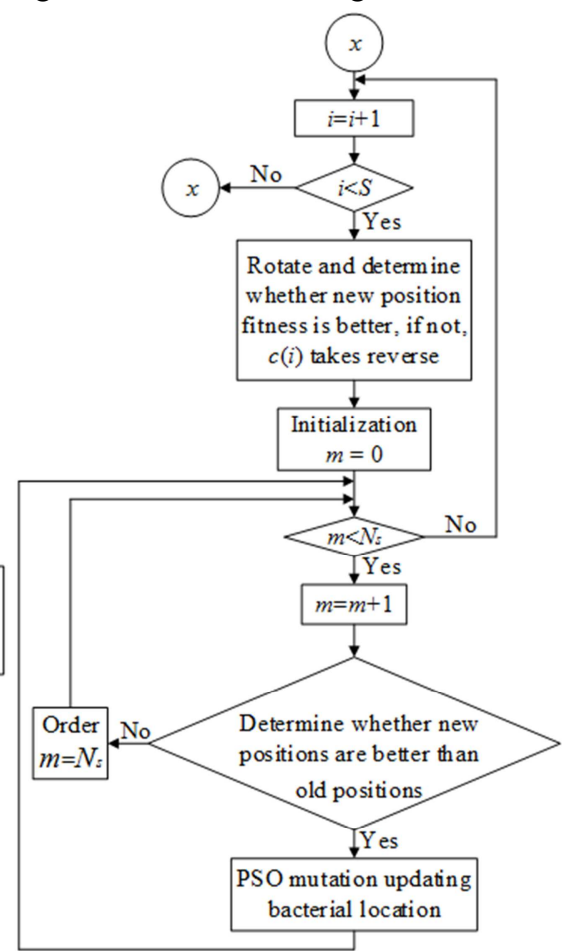

Figure 3. Flow of PSO-BFA algorithm. 


\section{Simulation Experiment}

The effectiveness of this method is studied by Matlab/Simulink simulation. The MPPT control system based on PSO-BFA is shown in Figure 4. Using Boost voltage regulating circuit to adjust voltage, the principle of realizing MPPT is that equivalent resistance of battery equals to photovoltaic array resistance. The resistance of PV array is influenced by ambient temperature and illumination. Boost circuit changes the battery resistance by changing duty cycle and tracking maximum power point. By changing the ratio of duty cycle through MPPT controller, the output voltage and algorithm search to match voltage value.

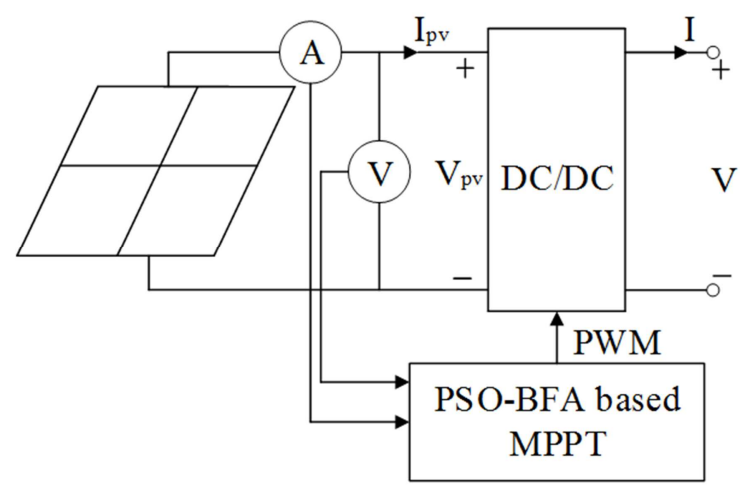

Figure 4. MPPT control system based on PSO-BFA.

Among them PV modules are parallel and parallel each other, and each component parallel a bypass diode. When a $\mathrm{PV}$ module is occluded, the component is subjected to reverse voltage, and the bypass diode can block inflow reverse current and avoid component heating damage. When the light is uniform, the temperature is $25^{\circ} \mathrm{C}$, and the light of $1000 \mathrm{~W} / \mathrm{m}^{2}$ is uniformly illuminated on four photovoltaic modules. If one of the PV modules is obscured, and the light intensity becomes $500 \mathrm{~W} / \mathrm{m}^{2}$. The other three modules remain the same, and the ambient temperature is the same, and there will be two peaks. As shown in Figure 5, the power of the two peaks is about $125 \mathrm{~W}$ and $94 \mathrm{~W}$.

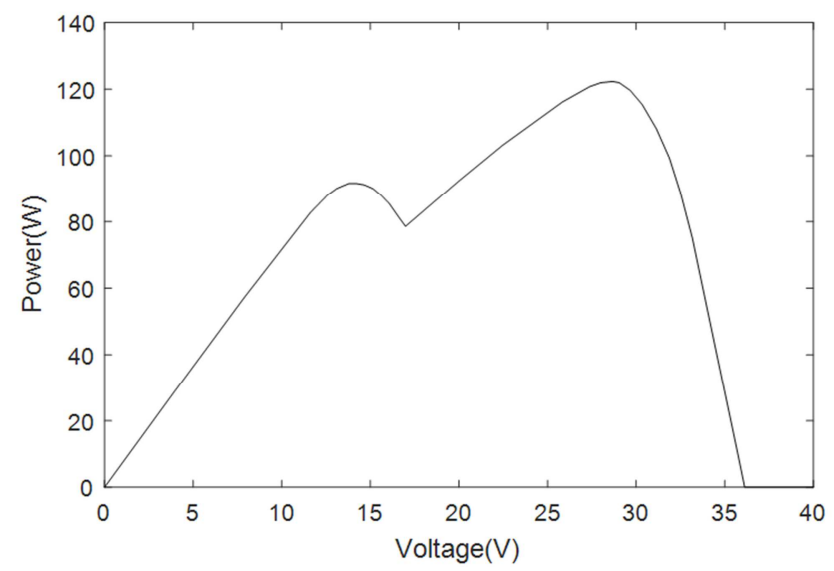

Figure 5. P-V output characteristics of photovoltaic array under partial shadow.

The MPPT is carried out by PSO-BFA, in which $\mathrm{N}_{P}$ takes 10 percent, $\mathrm{N}_{\mathrm{c}}$ takes 3 percent and $\mathrm{N}_{\mathrm{re}}$ takes 2 percent. The simulation results are shown in Figure 6. It can be seen from the figure that the maximum power of the search is about $125 \mathrm{~W}$ and the stabilization time is about $1.8 \mathrm{~s}$. Thus, it can be seen that the algorithm is fast and feasible.

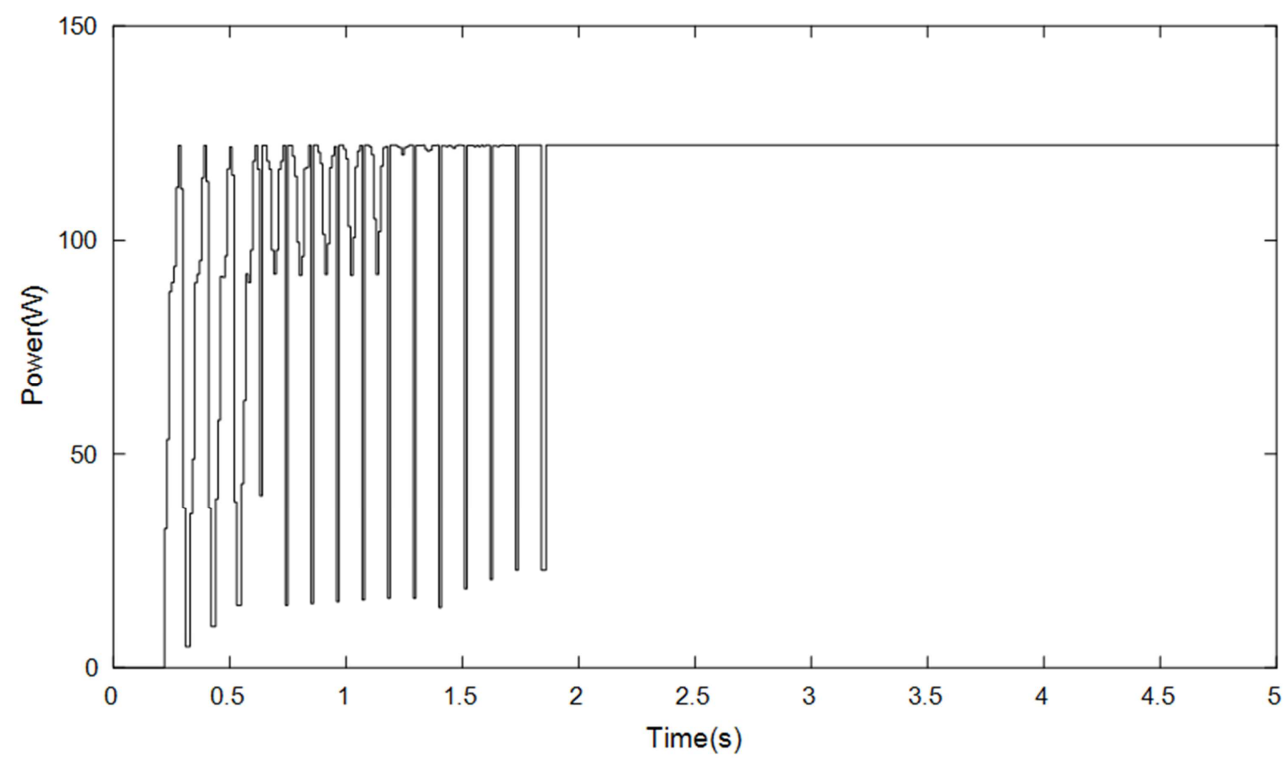

Figure 6. Simulation results of PSO-BFA.

\section{Conclusion}

In this paper, BFA and PSO algorithms are applied to the
MPPT of PV power generation. In order to improve the global searching ability of BFA, the particle swarm mutation operator is introduced to carry out global search. Then BFA is 
used to complete the local search. The simulation results show that the algorithm successfully realizes the search of the maximum power point in the multi-peak, and the convergence speed is fast. The application of this algorithm can effectively avoid falling into the local maximum value and improve the efficiency of PV power generation.

Through the analysis and simulation of the algorithm, the dynamic response of photovoltaic array is improved when the illumination intensity changes. The parameters are adjusted adaptively, and the better performance of photovoltaic system is obtained.

\section{References}

[1] D. Teshome, C. H. Lee, Y. W. Lin, and K. L. Lian, “A modified firefly algorithm for photovoltaic maximum power point tracking control under partial shading," IEEE J. Emerging Sel. Topics Power Electron., vol. 5, no. 2, pp. 661-671, Jun. 2017.

[2] R. Koad, A. F. Zobaa, and A. El Shahat, "A novel MPPT algorithm basedon particle swarm optimisation for photovoltaic systems," IEEE Trans. Sustainable Energy, vol. 8, no. 2, pp. 468-476, Apr. 2017.

[3] S. Mohanty, B. Subudhi, and P. K. Ray, "A new mppt design using grey wolf optimization technique for photovoltaic system under partial shading conditions," IEEE Trans. Sustain. Energy, vol. 7, no. 1, pp. 181-188, Jan.2016.

[4] Oliveira, F. M., Silva, S. A. O., Durand, F. R., et al.: 'Grid-tied photovoltaic system based on PSO MPPT technique with active power line conditioning',IET Power Electron., 2016, 9, (6), pp. $1180-1191$
[5] Renaudineau, H., Donatantonio, F., Fontchastagner, J., et al.: 'A PSO-based global MPPT technique for distributed PV power generation', IEEE Trans. Ind. Electron., 2015, 62, (2), pp. $1047-1058$

[6] Sérgio, A. O, S., Leonardo, P, S., Fernando M, O., et al.: 'Feed-forward DC-bus control loop applied to a single-phase grid-connected PV system operating with PSO-based MPPT technique and active power-line conditioning', IET Renew. Power Gener., 2017, Vol. 11 Iss. 1, pp. 183-193

[7] A. Ramyar, H. Iman-Eini, and S. Farhangi,“Global maximum powerpoint tracking method for photovoltaic arrays under partial shading conditions,"IEEE Trans. Ind. Electron., vol. 64, no. 4, pp. 2855-2864, Apr. 2017.

[8] M. Seyedmahmoudian et al., "Simulation and hardware implementation of new maximum powerpoint tracking technique for partially shaded PV system using hybrid DEPSO method," IEEE Trans. Sustain. Energy, vol. 6, no. 3, pp. 850 862, Jul. 2015.

[9] Nishant, K., Ikhlaq H., Bhim S., Bijaya, K. P., "Single sensor based MPPT for partially shaded solar photovoltaic by using human psychology optimisation algorithm," IET Gener. Transm. Distrib., 2017, Vol. 11 Iss. 10, pp. 2562-2574

[10] Mohanty, S., Subudhi, B., Ray, P. K.: 'A new MPPT design using grey wolf optimization technique for photovoltaic system under partial shading conditions', IEEE Trans. Sustain. Energy, 2016, 7, (1), pp. 181-188

[11] Neeraja Krishnakumar, Rini Venugopalan, N. Rajasekar, "Bacterial Foraging Algorithm based parameter estimation of solar PV model," International Conference on Microelectronics, Communication and Renewable Energy, 2013,pp. 1-6 